DESY-03-037

hep-ph/0303183

\title{
Flavor violation and warped geometry
}

\author{
Stephan J. Huber ${ }^{1}$ \\ Deutsches Elektronen-Synchrotron DESY, Hamburg, Germany
}

\begin{abstract}
Extra dimensions have interesting consequences for flavor physics. We consider a setup where the standard model fermions and gauge fields reside in the bulk of a warped extra dimension. Fermion masses and mixings are explained by flavor dependent fermion locations, without relying on hierarchical Yukawa couplings. We discuss various flavor violating processes induced by (Kaluza-Klein) gauge boson exchange and non-renormalizable operators. Experimental constraints are satisfied with a Kaluza-Klein scale of about 10 $\mathrm{TeV}$. Some processes, such as muon-electron conversion, are within reach of next generation experiments.
\end{abstract}

\footnotetext{
${ }^{1}$ stephan.huber@desy.de
} 


\section{Introduction}

Models with extra dimensions have attracted great attention in recent years as they offer new perspectives on challenging problems in modern physics. It was demonstrated by Randall and Sundrum that a small but warped extra dimension provides an elegant solution to the gauge hierarchy problem [1] (see also [2]). The fifth dimension is an $S_{1} / Z_{2}$ orbifold with an $\mathrm{AdS}_{5}$ geometry of curvature $k$, bordered by two 3-branes with opposite tensions and separated by distance $R$. The red shift induced by the AdS warp factor $\Omega=e^{-\pi k R}$ generates an exponential hierarchy between the energy scales on the two branes. If the brane separation is $k R \simeq 11$, the scale on the negative tension brane is of $\mathrm{TeV}$-size, while the scale on the other brane is of order $M_{\mathrm{Pl}}$. The AdS curvature $k$ and the 5D Planck mass $M_{5}$ are assumed to be of order $M_{\mathrm{Pl}}$. Gravity is weak at the TeV-brane because the zero mode corresponding to the $4 \mathrm{D}$ graviton is localized at the positive tension brane (Planck-brane).

In contrast to the original proposal [1] we take the standard model (SM) fermions and gauge bosons as bulk fields. In the non-supersymmetric framework we are studying, the Higgs field has to be confined to the TeV-brane in order to preserve the solution to the gauge hierarchy problem [3,4]. Our motivation is twofold. With the SM fermions residing in the 5-dimensional bulk, the hierarchy of quark and lepton masses can be related to a higher dimensional geography [5,6]. Different fermion flavors are localized at different positions in the extra dimension or, more precisely, have different wave functions. The fermion masses are in direct proportion to the overlap of their wave functions with the Higgs field [7]. Also the CKM mixing can be explained along these lines. A second point is that at the TeV-brane nonrenormalizable operators, now only TeV-scale suppressed, are known to induce rapid proton decay, large neutrino masses and flavor violating interactions. Because of the warp factor, the effective cut-off scale varies along the extra dimension. If the quarks and leptons are localized towards the Planck-brane in the extra dimension, the suppression scales of dangerous operators can be significantly enhanced $[5,6]$. Small Majorana neutrino masses can then arise from dimension-five interactions, without introducing new degrees of freedom [8]. The atmospheric and solar neutrino anomalies can be satisfactorily resolved. Alternatively, Dirac neutrino masses can be generated by a coupling to right-handed neutrinos in the bulk $[9,10]$.

Fields living in the 5D bulk can be expanded as a tower of Kaluza-Klein (KK) states. Electroweak observables, in particular the weak mixing angle and gauge boson masses, require the KK excitations of SM particles to be heavier than about $10 \mathrm{TeV}[4,11,12]$. A small hierarchy separates the weak and KK scales. In the presence of brane-localized kinetic terms the bound on the KK scale may be somewhat relaxed [13], but we are not considering this possibility in the following. For fermions confined to the TeV-brane, the electroweak constraints have been analyzed in ref. [14].

In this paper we address the issue of flavor violation in the warped SM which is an 
immediate consequence of our approach to the fermion mass problem. Some aspects of this topic have already been discussed in the literature [5,6,12,15-18]. The crucial connection to the issue of fermion masses, however, has not been thoroughly investigated so far. This connection allows us to obtain more reliable predictions for the flavor violating rates. Moreover, we are including lepton flavor violating processes which have not yet been considered in context of the warped SM. Some results have already been presented in ref. [19].

In the next section we summarize some results on bulk fermions and gauge bosons in a warped background. In section 3 we are using the observed fermion masses and mixings to determine the locations of the various fermion flavors in the extra dimension. The fermion mass pattern can be accommodated without relying on hierarchical Yukawa couplings. In section 4 we discuss how the mixing between different KK levels leads to a non-unitary CKM matrix. Flavor violation by (KK) gauge boson exchange, being a natural consequence of our setup, is studied in section 5. We find that with a $\mathrm{KK}$ scale of $10 \mathrm{TeV}$ the model is consistent with the experimental constraints, while some processes, such as muon-electron conversion, are within reach of next generation experiments. In section 6 we show that contributions from non-renormalizable operators to flavor violating processes are naturally within experimental bounds, while proton decay cannot by adequately suppressed. Finally, in section 7 we conclude.

\section{Bulk fields in a warped background}

We assume that the standard model gauge bosons and fermions reside in the bulk of the warped 5D space-time [1]

$$
d s^{2}=e^{-2 \sigma(y)} \eta_{\mu \nu} d x^{\mu} d x^{\nu}+d y^{2},
$$

where $\sigma(y)=k|y|$. The 4-dimensional metric is $\eta_{\mu \nu}=\operatorname{diag}(-1,1,1,1)$ and $y$ denotes the fifth coordinate. The AdS curvature $k$ is related to the bulk cosmological constant and the brane tensions. To set the notation let us briefly review some properties of gauge and fermion fields in a slice of $\mathrm{AdS}_{5}$.

A gauge field propagating in a curved background with metric $G^{M N}$ is described by the equation of motion

$$
\frac{1}{\sqrt{-G}} \partial_{M}\left(\sqrt{-G} G^{M N} G^{R S} F_{N S}\right)-M_{A}^{2} G^{R S} A_{S}=0 .
$$

The mass term $M_{A}^{2}$ arises from spontaneous symmetry breaking and is present only for the weak gauge bosons. Since the Higgs field is localized at the TeV-brane, we have

$$
M_{A}^{2}=\frac{1}{2}\left(g^{(5)}\right)^{2} v_{0}^{2} \delta(y-\pi R),
$$


where $g^{(5)}$ is the 5D gauge coupling. The Higgs vev $v_{0}$ is expected to be of order $M_{\mathrm{Pl}}$. Imposing the gauge $A_{5}=0$, we decompose the $5 \mathrm{D}$ fields as

$$
A_{\mu}(x, y)=\frac{1}{\sqrt{2 \pi R}} \sum_{n=0}^{\infty} A_{\mu}^{(n)}(x) f_{n}^{A}(y) .
$$

Inserting the metric (2.1) and the decomposition (2.4) into the equation of motion (2.2), the wave functions $f_{n}^{A}$ have to satisfy

$$
\left(\partial_{y}^{2}-2 \sigma^{\prime} \partial_{y}-M_{A}^{2}+e^{2 \sigma} m_{n}^{2}\right) f_{n}^{A}=0,
$$

where $\sigma^{\prime}=d \sigma / d y$. This equation is solved by [20]

$$
f_{n}^{A}(y)=\frac{e^{\sigma}}{N_{n}}\left[J_{1}\left(\frac{m_{n}}{k} e^{\sigma}\right)+b^{A}\left(m_{n}\right) Y_{1}\left(\frac{m_{n}}{k} e^{\sigma}\right)\right] .
$$

The spectrum of KK masses $m_{n}$ and the coefficients $b^{A}$ are determined by the boundary conditions of the wave functions at the branes. The localized mass term $M_{A}^{2}$ only affects the boundary condition at the TeV-brane $[4,11]$. The normalization constants are fixed by

$$
\frac{1}{2 \pi R} \int_{-\pi R}^{\pi R} d y f_{m}^{A}(y) f_{n}^{A}(y)=\delta_{m n} .
$$

In the case $M_{A}^{2}=0$ eq. (2.2) admits a constant zero mode solution [20]. The excited states are localized towards the TeV-brane and have TeV-scale KK masses $m_{n} \sim n \pi k e^{-\pi k R}$. In the presence of the mass term $M_{A}^{2}$, the zero mode acquires a mass

$$
m_{0}^{2}=\left(g^{(5)}\right)^{2} v_{0}^{2} e^{-2 \pi k R} /(2 \pi R)+\mathcal{O}\left(\left(g^{(5)}\right)^{4} v_{0}^{4} e^{-4 \pi k R} / M_{K K}^{2}\right),
$$

where $M_{K K}=m_{1}$ denotes the KK scale. The order $1 / M_{K K}^{2}$ corrections are related to a dip in the zero mode wave function caused by the boundary mass term [4]. Compared to the $4 \mathrm{D}$ standard model they induce a tree-level shift of the $\mathrm{W}$ and $\mathrm{Z}$ boson mass ratio relative to its $4 \mathrm{D}$ standard model value. In refs. [4, 11, 12] it was shown that the electroweak precision data thus implies the constraint $M_{K K} \gtrsim 10$ $\mathrm{TeV}$. If the boundary mass term was not included in the KK reduction, but evaluated with the $M_{A}^{2}=0$ wave functions, the order $1 / M_{K K}^{2}$ effects would show up in the $4 \mathrm{D}$ effective action as mixings between the KK states.

The equation of motion of a fermion in curved space-time reads

$$
E_{a}^{M} \gamma^{a}\left(\partial_{M}+\omega_{M}\right) \Psi+m_{\Psi} \Psi=0
$$

where $E_{a}^{M}$ is the fünfbein, $\gamma^{a}=\left(\gamma^{\mu}, \gamma^{5}\right)$ are the Dirac matrices in flat space, and

$$
\omega_{M}=\left(\frac{1}{2} e^{-\sigma} \sigma^{\prime} \gamma_{5} \gamma_{\mu}, 0\right)
$$


is the spin connection induced by the metric (2.1). Fermions have two possible transformation properties under the $Z_{2}$ orbifold symmetry, $\Psi(-y)_{ \pm}= \pm \gamma_{5} \Psi(y)_{ \pm}$. Thus, $\bar{\Psi}_{ \pm} \Psi_{ \pm}$is odd under $Z_{2}$, and the Dirac mass term, which is odd as well, can be parametrized as $m_{\Psi}=c \sigma^{\prime}$. The Dirac mass should therefore originate from the coupling to a $Z_{2}$ odd scalar field which acquires a vev. On the other hand, $\bar{\Psi}_{ \pm} \Psi_{\mp}$ is even. Using the metric (2.1) one obtains for the left- and right-handed components of the Dirac spinor $[5,9]$

$$
\left[e^{2 \sigma} \partial_{\mu} \partial^{\mu}+\partial_{5}^{2}-\sigma^{\prime} \partial_{5}-M_{f}^{2}\right] e^{-2 \sigma} \Psi_{L, R}=0,
$$

where $M_{f}^{2}=c(c \pm 1) k^{2} \mp c \sigma^{\prime \prime}$ and $\Psi_{L, R}= \pm \gamma_{5} \Psi_{L, R}$.

Decomposing the $5 \mathrm{D}$ fields as

$$
\Psi\left(x^{\mu}, y\right)=\frac{1}{\sqrt{2 \pi R}} \sum_{n=0}^{\infty} \Psi^{(n)}\left(x^{\mu}\right) e^{2 \sigma} f_{n}(y)
$$

one ends up with a zero mode wave function $[5,9]$

$$
f_{0}(y)=\frac{e^{-c \sigma}}{N_{0}}
$$

and a tower of KK excited states

$$
f_{n}(y)=\frac{e^{\sigma / 2}}{N_{n}}\left[J_{\alpha}\left(\frac{m_{n}}{k} e^{\sigma}\right)+b_{\alpha}\left(m_{n}\right) Y_{\alpha}\left(\frac{m_{n}}{k} e^{\sigma}\right)\right] .
$$

The order of the Bessel functions is $\alpha=|c \pm 1 / 2|$ for $\Psi_{L, R}$. The spectrum of KK masses $m_{n}$ and the coefficients $b_{\alpha}$ are determined by the boundary conditions at the branes $[5,9]$. The normalization constants follow from

$$
\frac{1}{2 \pi R} \int_{-\pi R}^{\pi R} d y e^{\sigma} f_{m}(y) f_{n}(y)=\delta_{m n}
$$

Because of the orbifold symmetry, the zero mode of $\Psi_{+}\left(\Psi_{-}\right)$is a left-handed (right-handed) Weyl spinor. For $c>1 / 2(c<1 / 2)$ the fermion is localized near the boundary at $y=0(y=\pi R)$, i.e. at the Planck- (TeV-) brane (see also fig. 1 ).

The zero modes of leptons and quarks acquire masses from their coupling to the Higgs field

$$
\int d^{4} x \int d y \sqrt{-G} \lambda_{i j}^{(5)} H \bar{\Psi}_{i+} \Psi_{j-} \equiv \int d^{4} x m_{i j} \bar{\Psi}_{i L}^{(0)} \Psi_{j R}^{(0)}+\cdots
$$

where $\lambda_{i j}^{(5)}$ are the 5D Yukawa couplings. The 4D Dirac masses are given by

$$
m_{i j}=\int_{-\pi R}^{\pi R} \frac{d y}{2 \pi R} \lambda_{i j}^{(5)} H(y) f_{0 i L}(y) f_{0 j R}(y)=\frac{l_{i j} v_{0}}{\pi k R} f_{0 i L}(\pi R) f_{0 j R}(\pi R) .
$$


In the second step we have used $H(y)=v_{0} \delta(y-\pi R) / k$ and introduced the dimensionless couplings $l_{i j}=\lambda_{i j}^{(5)} \sqrt{k}$.

The gauge interaction between bulk gauge bosons and fermions, $g^{(5)} \bar{\Psi} i E_{a}^{M} \gamma^{a} A_{M} \Psi$, induces the effective $4 \mathrm{D}$ couplings [5]

$$
g_{i j n}=\frac{g^{(5)}}{(2 \pi R)^{3 / 2}} \int_{-\pi R}^{\pi R} e^{\sigma} f_{i}(y) f_{j}(y) f_{n}^{A}(y) d y
$$

between the different KK levels. In the case of a massless gauge boson $\left(f_{0}^{A}(y) \equiv 1\right)$ this integral reduces to the normalization condition of fermions (2.15), and one finds $g_{i j 0}=\delta_{i j} g^{(5)} / \sqrt{2 \pi R}$. For a massive gauge field the gauge coupling of the zero mode to $\mathrm{TeV}$-brane fermions is somewhat reduced due to the dip in its wave function [4]. In the SM this effect leads, for instance, to smaller gauge couplings of $\mathrm{W}$ and $\mathrm{Z}$ bosons relative to that of the photon. In order to keep these corrections within experimental bounds we derived in ref. [11] the constraint $M_{K K} \gtrsim 60 \mathrm{TeV}$ if the fermions reside on the TeV-brane. For fermions localized towards the Planck-brane this constraint becomes weaker than the $10 \mathrm{TeV}$ bound from the gauge boson masses discussed before.

Combining eqs. (2.8) and (2.18), we can determine the Higgs vev required to provide the measured gauge boson masses. To keep the electroweak corrections small enough, we take $M_{K K}=10 \mathrm{TeV}$. Assuming $k=M_{\mathrm{Pl}}$ we find $k R=10.83$ and $v_{0}=0.043 M_{\mathrm{Pl}}$. Thus, there is still a small hierarchy of about twenty between the fundamental scale and the Higgs vev.

\section{Quark and lepton masses}

The fermion masses crucially depend on the overlap of the fermion wave functions and the Higgs profile. They are functions of the 5D mass parameters of the leftand right-handed fermions, $c_{L}$ and $c_{R}$ respectively, which enter eq. (2.17). As the 5D Dirac mass, i.e. $c$ parameter increases, the fermion gets localized closer towards the Planck-brane. Its overlap with the Higgs profile at the TeV-brane is reduced, which is reflected in a smaller $4 \mathrm{D}$ fermion mass from electroweak symmetry breaking. Since the fermionic zero modes depend exponentially on the 5D mass parameters, the large hierarchy of charged fermion masses can be generated from $c$ parameters of order unity $[5,6]$. If right-handed neutrinos are introduced in the bulk, sub-eV neutrino masses can be explained in the same manner $[9,10]$.

Building up the fermion mass matrices from eq. (2.17) requires also the specification of the 5D Yukawa couplings. Thus there are considerably more independent parameters in the model than there are observable fermion masses and mixings. Relating the measured fermion properties to their locations in the extra dimension needs therefore additional assumptions. In ref. [6] it was assumed that the 5D Yukawa couplings are of order unity, and the fermion mass pattern is solely due to the different locations. Additionally, the fermions were localized as closely as 
possible towards the Planck-brane in order to maximally suppress the impact of non-renormalizable operators on rare processes.

In the following we are using a somewhat different approach. While still assuming the 5D Yukawa couplings to be of order unity, we are looking for a set of $c$ parameters which "most naturally" accounts for the observed fermion masses and mixings. More precisely, we are taking random 5D Yukawa couplings and require the averaged fermion properties to fit the experimental data. Similar methods have been used to study models where the fermion mass pattern is due to (approximately) conserved charges [21]. There the role of the fermion locations is taken by a set of Higgs fields.

Let us first focus on the quark sector. At the scale of $10 \mathrm{TeV}$, where we are matching our model to the observational data, the (running) quark masses are

$$
\begin{aligned}
& m_{u}=0.7-2.3 \mathrm{MeV}, \quad m_{c}=420-540 \mathrm{MeV}, \quad m_{t}=140-148 \mathrm{GeV} \\
& m_{d}=1.4-4.2 \mathrm{MeV}, \quad m_{s}=28-80 \mathrm{MeV}, \quad m_{b}=2.1-2.3 \mathrm{GeV} .
\end{aligned}
$$

We have used one loop renormalization group equations [22] to run the quark masses given in ref. [23] from the scale $M_{Z}$ to $10 \mathrm{TeV}$. The running reduces the quark masses by about 20 percent. The moduli of the CKM matrix are given by [24]

$$
\left|V_{\mathrm{CKM}}\right|=\left(\begin{array}{ccc}
0.9741-0.9756 & 0.219-0.226 & 0.0025-0048 \\
0.219-0.226 & 0.9732-0.9748 & 0.038-0.044 \\
0.004-0.014 & 0.037-0.044 & 0.9990-0.9993
\end{array}\right)
$$

A convention independent measure of $\mathrm{CP}$ violation is the Jarlskog invariant which experimentally is found to be [24]

$$
J=(3 \pm 0.3) \times 10^{-5}
$$

Assuming non-hierarchical 5D Yukawa couplings, the fermion mass matrix (2.17) leads to a product-like structure

$$
M \sim\left(\begin{array}{ccc}
a_{1} b_{1} & a_{1} b_{2} & a_{1} b_{3} \\
a_{2} b_{1} & a_{2} b_{2} & a_{2} b_{3} \\
a_{3} b_{1} & a_{3} b_{2} & a_{3} b_{3}
\end{array}\right)
$$

where $a_{i}$ and $b_{i}$ are given by the fermion wave functions $f_{0 i L, R}(\pi R)$. As a function of the fermion location there is a slow increase $f_{0}(\pi R) \propto \sqrt{1 / 2-c}$ for $c<1 / 2$, and an exponential suppression $f_{0}(\pi R) \propto \exp (-c \pi k R)$ for $c>1 / 2$. Note that some nonequal Yukawa couplings are needed to render the mass matrix (3.22) non-singular. If the mass matrix is diagonalized by $U_{L} M U_{R}^{\dagger}$, the left- and right-handed mixings are typically of order $U_{L, i j} \sim a_{i} / a_{j}$ and $U_{R, i j} \sim b_{i} / b_{j}$, respectively. Fermions which have similar positions ( $c$ parameters) have large mixings. The mass matrix (3.22) predicts the approximate relation $U_{13} \sim U_{12} U_{23}$ between the mixing angles, which for the observed CKM matrix (3.20) is satisfied up to a factor of about two. 


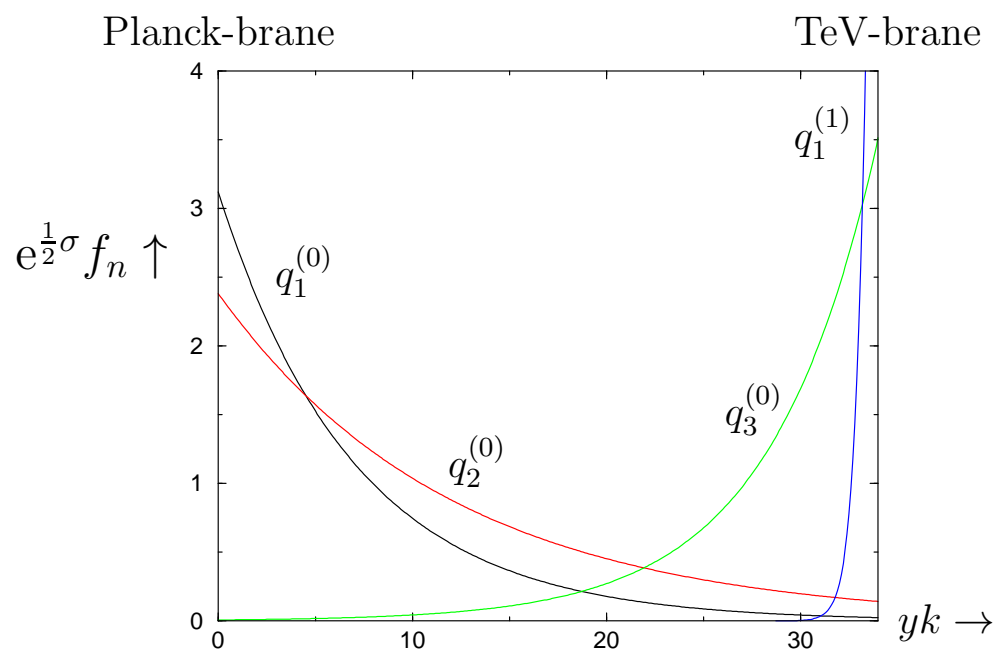

Figure 1: The wave functions of the left-handed quark zero modes $q_{i}^{(0)}$ and the first KK state, $q_{1}^{(1)}$, of $Q_{1}$ for the parameters of eq. (3.24).

In our numerical analysis we generate $N=25000$ random sets of up and downtype Yukawa couplings and diagonalize the emerging mass matrices (2.17). We require the averaged fermion masses and mixings to fit the experimental data, using the logarithmic average of a quantity $X$

$$
\langle X\rangle=\exp \left(\sum_{i}^{N} \frac{\ln \left(X_{i}\right)}{N}\right) .
$$

Taking $2 / 3<\left|l_{i j}\right|<4 / 3$ and random phases from 0 to $2 \pi$, we find the "most natural" locations

$$
\begin{array}{lll}
c_{Q 1}=0.643, & c_{D 1}=0.643, & c_{U 1}=0.671 \\
c_{Q 2}=0.583, & c_{D 2}=0.601, & c_{U 2}=0.528 \\
c_{Q 3}=0.317, & c_{D 3}=0.601, & c_{U 3}=-0.460 .
\end{array}
$$

The wave functions of the left-handed quark zero modes and the first excited state of $Q_{1}$ are shown in fig. 1. We fix the relative positions of $Q_{1}$ and $Q_{2}$ by fitting $\left|V_{u s}\right|$, while $\left|V_{c b}\right|$ determines the relative positions of $Q_{2}$ and $Q_{3}$. The Jarlskog invariant $J=\operatorname{Im}\left(V_{c s} V_{u s}^{*} V_{u d} V_{c d}^{*}\right)[25]$ and $\left|V_{u b}\right|$ are then fixed as well. Note that the the CKM mixings are determined by the locations of the left-handed quarks. We then use the locations of the right-handed quarks to fit the quark masses. Taking the quark locations (3.24), we find for the averages

$$
\begin{array}{lll}
m_{u}=2.0 \mathrm{MeV}, & m_{c}=506 \mathrm{MeV}, & m_{t}=144 \mathrm{GeV}, \\
m_{d}=4.0 \mathrm{MeV}, & m_{s}=58 \mathrm{MeV}, & m_{b}=2.2 \mathrm{GeV} \\
\left|V_{u s}\right|=0.222, & \left|V_{c b}\right|=0.040, & \left|V_{u b}\right|=0.0088 \\
J=3.1 \times 10^{-5} & &
\end{array}
$$


While the prediction of $J$ agrees well with the experimental value (3.21), $\left|V_{u b}\right|$ is found to be about two times too large (3.20). As discussed above this discrepancy is a consequence of the mass pattern (3.22).

The quark locations of eq. (3.24) are not a unique solution. Moving all the lefthanded quarks in such a way that the related factors $a_{i}$ in (3.22) are changed by a common factor, can be compensated by an appropriate change in the locations of the right-handed quarks. So in fact there is a one parameter family of solutions, labeled, for instance, by $\delta c_{Q 1}$. However, if some of the light quarks are localized towards the TeV-brane $(c<1 / 2)$, large deviations from electroweak precision variables drive the KK scale far above $10 \mathrm{TeV}$ [11]. In section 5 we will see that in this case flavor violation is greatly enhanced. Since $c_{U 2}$ is already close to $1 / 2$, we cannot move the right-handed quarks much closer to the TeV-brane. This would also require to localize the right-handed top quark extremely close towards the TeV-brane by $c_{U 3} \lesssim-2$. On the other hand, the $Q_{i}$ cannot be localized much closer towards the TeV-brane without getting too large modifications of the left-handed bottom couplings. Thus we end up with the quite constrained range of $-0.01 \lesssim \delta c_{Q 1} \lesssim 0.02$. The quarks can be localized closer towards the Planck-brane if the 5D Yukawa couplings are increased by a common factor. Increasing them by a factor of 10 , for instance, corresponds to $\delta c_{Q 1}=0.037$. However, very large Yukawa couplings $l \gg 1$ introduce a new and unexplained hierarchy in the model. Taking Yukawa couplings $l \ll 1$ would mean to move the quarks closer towards the TeV-brane which worsens the electroweak fit. The quark locations also depend on $k / M_{\mathrm{Pl}}$ which we take to be one.

The experimental errors (3.19) and (3.20) translate into uncertainties in the fermion locations. For instance, from $\left|V_{c b}\right|$ we can fix the position of $Q_{3}$ relative to $Q_{2}$ only up to an error of $-0.02 \lesssim \Delta c \lesssim 0.03$. The position of $Q_{1}$ relative to $Q_{2}$ is determined quite accurately up to about $-6 \times 10^{-4} \lesssim \Delta c \lesssim 5 \times 10^{-4}$ by $\left|V_{u s}\right|$. For the right-handed quarks the errors induced by the quark masses range from $\Delta c \approx \pm 8 \times 10^{-4}$ for $D_{3}$ to $\Delta c \approx \pm 0.03$ for $U_{3}$. More precisely, these are errors on the relative positions of left- and right-handed quarks, for instance, $c_{Q 3}-c_{U 3}$ in the case of the top. Note that positions $c<1 / 2$ have larger errors since in this range the fermion mass does not depend exponentially but as $\sqrt{1 / 2-c}$ on the fermion location.

The statistical approach provides us with distributions of fermion properties rather than with their precise values. This adds further uncertainties to the fermion locations. In fig. 2a we present the distribution of $\ln \left(m_{u}\right)$ which shows a half width of about 1.1. Requiring that, with a different location, the average value of $\ln \left(m_{u}\right)$ still lies within this half width range translates into an uncertainty of $-0.017 \lesssim \Delta c_{U 1} \lesssim 0.019$. In table 1 we have summarized the statistical and experimental uncertainties in the quark locations. Fig. 2b shows the distribution of $\ln \left(\left|V_{u b}\right|\right)$ which overlaps considerably with the experimentally favored range. Despite the factor of two deviation in the average $\left\langle\left|V_{u b}\right|\right\rangle$, there is an acceptably good fit to the data once the statistical variations are taken into account. 

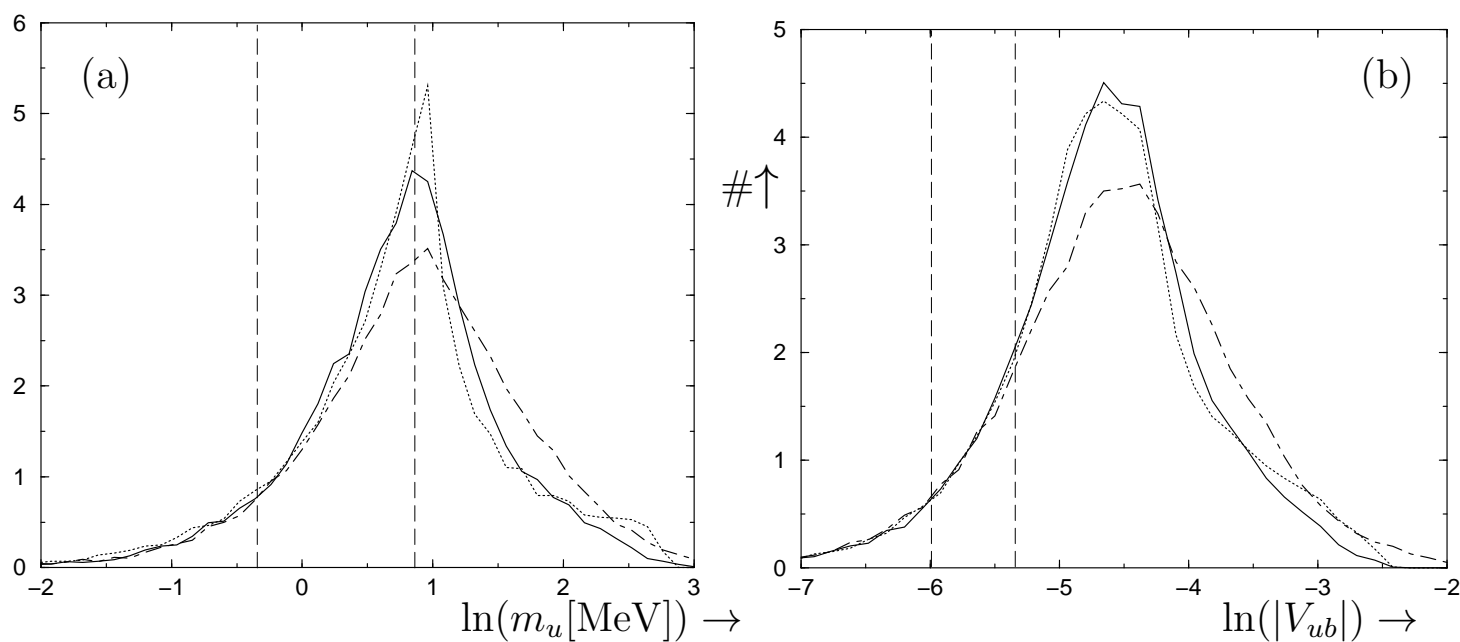

Figure 2: Logarithmic distributions of $m_{u}$ (a) and $\left|V_{u b}\right|$ (b) for Yukawa coupling distributions $2 / 3<\left|l_{i j}\right|<4 / 3$ (solid lines), $0<\left|l_{i j}\right|<2$ (dashed dotted lines) and $\left|l_{i j}\right|=1$ (solid lines). The vertical dashed lines indicate the experimental uncertainties.

Our results depend only weakly on the range taken for the moduli of the 5D couplings $l_{i j}$. In fig. 2 we present the distributions of $m_{u}$ and $\left|V_{u b}\right|$ additionally for the case $0<\left|l_{i j}\right|<2$ and for pure phase Yukawa couplings $\left|l_{i j}\right|=1$. In the context of flat extra dimensions pure phase Yukawa couplings have recently been investigated in ref. [26]. We find that a wider range of $\left|l_{i j}\right|$ leads only to a slight broadening in the distributions of fermion properties. The induced shift in the average values is always much smaller than the width of the distributions. For instance, we find an average $m_{u}=2.3(2.0) \mathrm{MeV}$ in the case $0<\left|l_{i j}\right|<2\left(\left|l_{i j}\right|=1\right)$. This behavior stresses that the fermion mass pattern is indeed determined by the fermion locations, not by accidental properties of the 5D Yukawa matrices. Note that the robustness of our results concerning different ranges of $\left|l_{i j}\right|$ is due to the complex phases in the $5 \mathrm{D}$ Yukawa couplings. For real values of $l_{i j}$ there is a high probability of cancellations in the mass matrices. This leads to multiple peaks in the distributions of fermion masses and mixings for a narrow range of $\left|l_{i j}\right|$.

Let us close this section with a brief discussion of the lepton sector. To determine the lepton locations we have to take into account neutrino masses and

\begin{tabular}{|c||c|c|c|c|c|c|c|c|c|}
\hline & $Q_{1}$ & $Q_{2}$ & $Q_{3}$ & $D_{1}$ & $D_{2}$ & $D_{3}$ & $U_{1}$ & $U_{2}$ & $U_{3}$ \\
\hline$c$ & 0.643 & 0.583 & 0.317 & 0.643 & 0.601 & 0.601 & 0.671 & 0.528 & -0.460 \\
\hline exp. & +0.0004 & +0.002 & +0.03 & +0.017 & +0.012 & +0.0008 & +0.017 & +0.004 & +0.03 \\
& -0.0006 & -0.003 & -0.02 & -0.008 & -0.006 & -0.0008 & -0.002 & -0.002 & -0.03 \\
\hline stat. & +0.022 & +0.029 & +0.13 & +0.016 & +0.006 & +0.004 & +0.019 & +0.006 & +0.50 \\
& -0.027 & -0.024 & -0.21 & -0.023 & -0.017 & -0.006 & -0.017 & -0.023 & -0.76 \\
\hline
\end{tabular}

Table 1: Experimental and statistical errors on the fermion locations of eq. (3.24). 
mixings. Neutrino masses can arise from different sources in the context of warped geometry. In one scenario sub-eV Dirac masses are generated by a coupling to right-handed neutrinos in the bulk $[9,10]$. The necessary tiny Yukawa couplings can naturally be generated if the right-handed neutrinos are localized closely towards the Planck-brane by taking $1.2 \lesssim c \lesssim 1.5$. Neutrino and charged fermion masses are then treated on the same footing. Alternatively, the dimension five interaction $(1 / Q) H H L L$ can induce small Majorana neutrino masses [8]. The suppression scale $Q$ of the non-renormalizable interaction depends on the position of the left-handed leptons $L$ in the extra dimension. This mechanism is minimal in the sense that it does not require to extend the standard model particle content.

As in the case of quarks, the locations of the left-handed states determine the observed fermion mixings. Large neutrino mixings require the neutrinos and thus the $\mathrm{SU}(2)$ lepton doublets to have similar positions $c_{L i}$

$$
\begin{array}{lll}
c_{L 1}=0.565, & c_{L 2}=0.565, & c_{L 3}=0.565, \\
c_{E 1}=0.764, & c_{E 2}=0.609, & c_{E 3}=0.509 .
\end{array}
$$

The right-handed positions $c_{E i}$ we fixed by requiring that with random Yukawa couplings the average charged lepton masses fit their observed values.

To suppress the matrix element $U_{e 3}$ in the neutrino mixing matrix it is favorable to separate the electron doublet somewhat from the muon and tau doublets [8]

$$
\begin{array}{lll}
c_{L 1}=0.631, & c_{L 2}=0.565, & c_{L 3}=0.565, \\
c_{E 1}=0.725, & c_{E 2}=0.594, & c_{E 3}=0.497 .
\end{array}
$$

Like in the case of quarks there is a one parameter family of degenerate locations if the left- and right-handed states are shifted against each other. The widths of the distributions allow to fix the locations only up to $\Delta c \approx \pm 0.01$. Taking the 5 D Yukawa couplings larger than one, we could shift the leptons closer towards the Planck-brane, as was done in ref. [8].

\section{Mixings of KK states and the unitarity of the CKM matrix}

So far we have only dealt with the zero modes of quarks and leptons. But the Yukawa interaction (2.16) also induces mixings between the zero modes and the vector-like excited states. In the following we show that this effect hardly modifies the conclusions we have reached above.

From the KK reduction of an $\mathrm{SU}(2)$ doublet quark $Q_{L}$ we obtain a left-handed zero mode $q_{L}^{(0)}$, corresponding to the SM quark, and an infinite tower of left- and right-handed KK excited states $q_{L}^{(n)}$ and $q_{R}^{(n)}$, where we omit flavor indices. The

$\mathrm{SU}(2)$ singlet up quarks decompose into the right-handed zero mode $u_{R}^{c(0)}$ and the 
KK excited states $u_{L}^{c(n)}$ and $u_{R}^{c(n)}$. After electroweak symmetry breaking the up quark mass matrix takes the form

$$
M_{U}=\left(\bar{u}_{L}^{(0)}, \bar{u}_{L}^{(1)}, \bar{u}_{L}^{c(1)}, \ldots\right)\left(\begin{array}{cccc}
m^{(0,0)} & 0 & m^{(0,1)} & \ldots \\
m^{(1,0)} & m_{Q, 1} & m^{(1,1)} & \ldots \\
0 & 0 & m_{U, 1} & \ldots \\
\vdots & \vdots & \vdots & \ddots
\end{array}\right)\left(\begin{array}{c}
u_{R}^{c(0)} \\
u_{R}^{(1)} \\
u_{R}^{c(1)} \\
\vdots
\end{array}\right)
$$

where we again suppress flavor indices, i.e. every entry represents a $3 \times 3$ matrix in flavor space. The masses $m^{(m, n)}$ arise from electroweak symmetry breaking. They are obtained by inserting the relevant wave functions into eq. (2.17). The $m_{Q, m}$ and $m_{U, m}$ denote the KK masses of the excited quark states. The zeros in (4.28) follow from the $Z_{2}$ orbifold properties of the wave functions. Analogous mass matrices arise for the down quarks and charged leptons. The wave functions of the the KK fermions are localized at the TeV-brane (see fig. 1). Their overlap with the Higgs is large and almost independent of the location of the zero mode. We obtain

$$
\begin{aligned}
m^{(0, n)} & =\frac{l v_{0}}{\pi k R} f_{0 L}(\pi R) f_{n R}(\pi R) \\
m^{(m, n)} & =\frac{l v_{0}}{\pi k R} f_{m L}(\pi R) f_{n R}(\pi R) \approx(-1)^{m+n} \cdot l \cdot 349 \mathrm{GeV}, \quad m, n \geq 1
\end{aligned}
$$

These masses are large compared to the corresponding zero mode masses.

The mass matrix (4.28) induces a mixing between $\mathrm{SU}(2)$ singlet and doublet states. This effect diminishes the weak charge of the left-handed quarks [16]. In the context of neutrinos this behavior was discussed in refs. [9, 10]. Ignoring flavor mixing for a moment, the singlet admixture in the left-handed zero mode is

$$
\sum_{n=1}^{\infty}\left(\frac{m^{(0, n)}}{m_{U, n}}\right)^{2} \approx\left(\frac{m^{(0,1)}}{m_{U, 1}}\right)^{2} \sum_{n=1}^{\infty} \frac{1}{n^{2}}=\left(\frac{m^{(0,1)}}{m_{U, 1}}\right)^{2} \frac{\pi^{2}}{6} .
$$

In the second step we approximated the KK spectrum by $m_{n} \approx n m_{1}$. Taking the quark positions of eq. (3.24) we obtain for the top quark a singlet admixture of $1.5 \times 10^{-4}$. For the other quark flavors the admixture is smaller than $10^{-6}$. The mixing between the $\mathrm{SU}(2)$ doublet zero mode and its $\mathrm{KK}$ excitations is even more suppressed and on the order of $\left(m^{(0,1)} m^{(1,1)}\right)^{2} / m_{Q, 1}^{4}$. Our neglect of the KK states in the discussion of quark and lepton masses in the previous section is therefore well justified.

The gauge couplings of fermions (2.18) are modified by KK mixing in the weak gauge boson sector $[4,11]$. The $\mathrm{W}$ and $\mathrm{Z}$ bosons couple somewhat weaker to fermions at the TeV-brane, while their KK states couple stronger $[5,20]$, as we show in fig. 3 . For $c>1 / 2$ the gauge coupling is almost independent of the precise location, and the coupling of the KK states is small, $g_{1} / g_{S M} \approx 0.19$. 

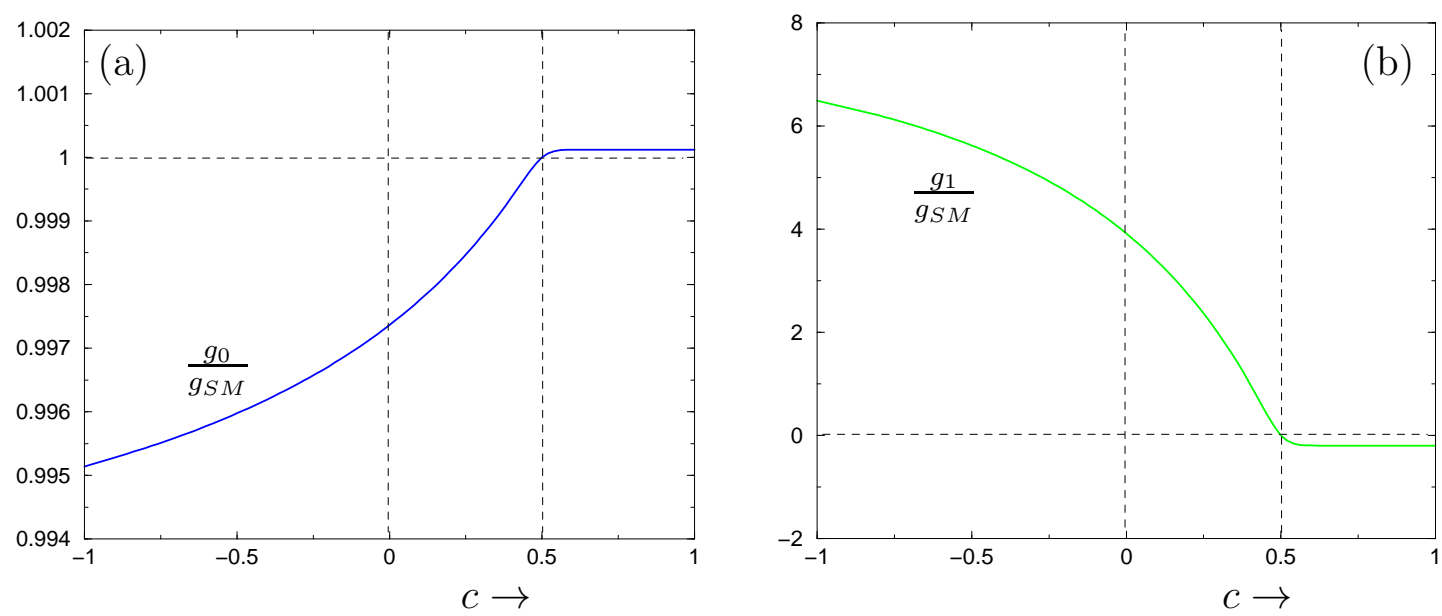

Figure 3: Gauge couplings of the $\mathrm{Z}$ boson (a) and its first KK state relative to the $\mathrm{SM}$ value as a function of the fermion location.

The charged current interaction of the KK fermions is described by an infinitely dimensional version of the CKM matrix

$$
\mathcal{V}=\mathcal{U}_{L, U} \mathcal{G U}_{L, D}^{\dagger}
$$

where $\mathcal{U}_{L}$ and $\mathcal{U}_{R}$ diagonalize the full fermion mass matrices (4.28). The flavor diagonal matrix $\mathcal{G}$ contains the gauge couplings of the various KK states to the W boson. We normalize $\mathcal{G}$ to the weak coupling of the muon as obtained from eq. (2.18) with the muon location of eq. (3.26). Since the weak gauge bosons are massive, they induce couplings between different KK levels. These couplings are suppressed by $M_{Z} / M_{K K}$ and numerically small. Note that in general even the full CKM matrix $\mathcal{V}$ is not unitary. Its truncation to the zero mode sector, $V$, governs the low energy charged current interaction. Three sources contribute to the non-unitarity of $V$ : the mixing of $\mathrm{SU}(2)$ doublet and singlet states, the truncation to the zero mode sector and the modification of the fermion gauge couplings. The last contribution is dominant, except for the third generation quarks. Defining $\Delta V_{i}=\sum_{j=1}^{3}\left|V_{i j}\right|^{2}-1$, we find with the quark locations of eq. (3.24) $\Delta V_{1} \approx 1 \cdot 10^{-5}, \Delta V_{2} \approx 2 \cdot 10^{-7}$ and $\Delta V_{3} \approx-3 \cdot 10^{-3}$. Again we have averaged over random sets of Yukawa couplings. The deviation from unitarity in the first generation is two orders of magnitude below the current experimental sensitivity [24]. Also the expectation of $\Delta V_{3}$ is small compared to the few percent precision in the weak charge of the top quark to be reached at the LHC [27].

Charged current interactions are also mediated by the KK states of the W boson. These corrections are suppressed by $\left(g_{1}^{2} / \delta g_{0}^{2}\right)\left(M_{W}^{2} / M_{K K}^{2}\right) \lesssim 0.1$ and therefore subleading. The inequality becomes satisfied for the top quark which is localized closest towards the TeV-brane. Only for fermions localized very closely to the TeV-brane these corrections become important. 


\section{$5 \quad$ Flavor violation}

The mechanisms we have discussed in the previous section in the context of charged current sector modify the neutral current interactions in a similar way. In analogy to eq. (4.30) we define the neutral current gauge couplings in the basis of mass eigenstates as

$$
\mathcal{X}_{L, R}^{\psi(n)}=\mathcal{U}_{L, R}^{\psi} \mathcal{G}_{L, R}^{\psi(n)} \mathcal{U}_{L, R}^{\psi \dagger}
$$

where the unitary matrices $\mathcal{U}_{L, R}^{\psi}$ diagonalize the full mass matrices, including the KK states of the fermion species $\psi=q_{L}, u_{R}, l_{L}$, etc. In ref. [19] we had not yet included KK mixing in the fermion sector. With $\mathcal{G}_{L, R}^{\psi n}$ we denote the coupling to the $n$th KK state of the Z boson as obtained from eq. (2.18). If the fermion families are localized at different positions in the extra dimension, the gauge couplings $\mathcal{G}_{L, R}^{\psi n}$ are non-universal in flavor space. These deviations in the fermion gauge couplings are too small to directly show up in flavor conserving processes at present accelerator experiments if the KK scale is about $10 \mathrm{TeV}$ [11]. However, the transformation to the mass eigenstates (5.31) induces flavor violating couplings which lead to interesting new phenomena. These flavor violating interactions are an immediate consequence of our approach to the problem of fermion masses. The phenomenology is similar to what happens in models with family non-universal Z' bosons, so we can adopt the formalism described, for instance, in ref. [28] with the complication of the KK mixing of fermions. In the gauge boson sector we work with mass eigenstates from the very beginning. There is no mixing of the $\mathrm{Z}$ boson and its KK states, instead the zero mode has flavor violating couplings as well.

Flavor violation as induced by the couplings (5.31) is driven by the non-universality $\delta g_{i j}$ in the couplings of different flavor states which are mixed by an angle $\theta_{i j}$

$$
\mathcal{X}_{i j} \approx \delta g_{i j} \sin \theta_{i j}
$$

Very different fermion locations on the one hand increase non-universality, on the other hand they lead to small mixing angles. Non-universality is larger for fermions localized towards the TeV-brane, $c<1 / 2$, as is shown in fig. 3 , and for the $\mathrm{Z}$ zero mode depends on the KK scale as $1 / M_{K K}^{2}$. Note that, different from the standard model, the right-handed mixings become physically relevant. The lefthanded mixings of quarks and leptons are naturally comparable to the observed CKM and neutrino mixing angles, respectively. This requirement fixed the lefthanded fermion locations in section 3. The locations of right-handed fermions we have used to fit the fermion masses. For the locations (3.24) and (3.27) the righthanded mixings are then predicted as

$$
\begin{array}{ll}
\left\langle\left|\mathcal{U}_{R, 1,2}^{u}\right|\right\rangle=0.017, \quad\left\langle\left|\mathcal{U}_{R, 2,3}^{u}\right|\right\rangle=0.073, & \left\langle\left|\mathcal{U}_{R, 1,3}^{u}\right|\right\rangle=0.0012, \\
\left\langle\left|\mathcal{U}_{R, 1,2}^{d}\right|\right\rangle=0.23, \quad\left\langle\left|\mathcal{U}_{R, 2,3}^{d}\right|\right\rangle=0.60, \quad & \left\langle\left|\mathcal{U}_{R, 1,3}^{d}\right|\right\rangle=0.23 \\
\left\langle\left|\mathcal{U}_{R, 1,2}^{e}\right|\right\rangle=0.016, \quad\left\langle\left|\mathcal{U}_{R, 2,3}^{e}\right|\right\rangle=0.057, \quad & \left\langle\left|\mathcal{U}_{R, 1,3}^{e}\right|\right\rangle=0.0016 .
\end{array}
$$


Again we have averaged over random sets of Yukawa couplings. The right-handed down quarks mix strongly with each other, while the mixings of up quarks and leptons are found to be small. For the alternative other set of lepton locations (3.26) the right-handed mixings are similarly small.

In the following we are using a KK scale of $10 \mathrm{TeV}$ which might be somewhat lowered by introducing brane-kinetic terms [13]. Note that the rates of flavor violating processes which we present below cannot be translated to that case by simple scaling with the appropriate powers of $M_{K K}$. Brane-kinetic terms modify the KK wave functions and thus possible non-universalities even if the KK scale is not changed.

\subsection{Lepton flavor violation}

Let us start the discussion of flavor violation with the lepton sector. In addition to the lepton locations (A) (3.26) and (B) (3.27) we are considering two modified settings

$$
\begin{array}{llll}
\left(\mathrm{A}^{\prime}\right): & c_{L 1}=0.585, & c_{L 2}=0.565, & c_{L 3}=0.545, \\
\left(\mathrm{~B}^{\prime}\right): & c_{L 1}=0.520, & c_{L 2}=0.200, & c_{L 3}=0.150
\end{array}
$$

to explore the dependence on the fermion locations. The right-handed positions are taken as in (A) and (B), respectively. In the set (A') we have shifted the lefthanded leptons by a small amount to induce some non-universality in the left-handed sector. The changes in the lepton masses and mixings remain within the widths of the statistical distributions. In (B') we have reduced the Yukawa couplings by a common factor of twenty, which is compensated by moving the left-handed leptons closer towards the TeV-brane where non-universality is especially large. We also separated somewhat the left-handed muon and tau states. Using these locations we find the following lepton flavor violation $\mathrm{Z}$ couplings

(A)

$$
\begin{array}{lllll}
\left\langle\left|\mathcal{X}_{L, 1,2}^{e(0)}\right|\right\rangle: & 2.3 \times 10^{-7} & 6.5 \times 10^{-7} & 3.7 \times 10^{-7} & 8.4 \times 10^{-5} \\
\left\langle\left|\mathcal{X}_{L, 2,3}^{e(0)}\right|\right\rangle: & 5.1 \times 10^{-7} & 1.2 \times 10^{-6} & 3.5 \times 10^{-7} & 3.2 \times 10^{-5} \\
\left\langle\left|\mathcal{X}_{L, 1,3}^{e(0)}\right|\right\rangle: & 2.3 \times 10^{-7} & 8.5 \times 10^{-7} & 2.2 \times 10^{-7} & 4.9 \times 10^{-5} \\
\left\langle\left|\mathcal{X}_{R, 1,2}^{e(0)}\right|\right\rangle: & 7.1 \times 10^{-9} & 9.7 \times 10^{-9} & 1.0 \times 10^{-8} & 7.7 \times 10^{-9} \\
\left\langle\left|\mathcal{X}_{R, 2,3}^{e(0)}\right|\right\rangle: & 7.6 \times 10^{-7} & 1.1 \times 10^{-6} & 2.3 \times 10^{-6} & 1.7 \times 10^{-6} \\
\left\langle\left|\mathcal{X}_{R, 1,3}^{e(0)}\right|\right\rangle: & 7.3 \times 10^{-9} & 8.5 \times 10^{-9} & 6.5 \times 10^{-8} & 4.6 \times 10^{-8}
\end{array}
$$

where we have included the zero modes and the first KK states of the leptons. Note that in the case (B') there is a large increase in the left-handed couplings compared to the cases with $c \gtrsim 1 / 2$. For $(\mathrm{A})$ the flavor violating left-handed couplings completely 
stem from fermion KK mixing since there is no non-universality among the lefthanded states. In the other cases fermion KK mixing is a subleading effect.

The couplings (5.35) induce lepton flavor violating decays of the Z boson with a branching ratio $\operatorname{Br}\left(Z \rightarrow l_{i} \bar{l}_{j}\right) \approx 0.29 \cdot\left(\left|\mathcal{X}_{L, i, j}^{e(0)}\right|^{2}+\left|\mathcal{X}_{R, i, j}^{e(0)}\right|^{2}\right)$. The largest rate occurs in scenario (B') with $(Z \rightarrow e \bar{\mu}) \approx 2 \times 10^{-9}$ which is still three orders of magnitude below the experimental bound of $1.7 \times 10^{-6}$ [24]. The other decay modes are even stronger suppressed. Thus there are no constraints on the model from lepton flavor violating $\mathrm{Z}$ decays.

The off-diagonal elements of $\mathcal{X}$ also lead to flavor violating decays of charged leptons. Tree-level exchange of a $\mathrm{Z}$ boson and its KK states induces the processes $l_{i} \rightarrow 3 l_{j}$ at a rate

$$
(\mathrm{A}) \quad\left(\mathrm{A}^{\prime}\right) \quad(\mathrm{B}) \quad\left(\mathrm{B}^{\prime}\right)
$$

$$
\begin{array}{rllll}
\operatorname{Br}(\mu \rightarrow \text { eеe }): & 5.2 \times 10^{-14} & 4.7 \times 10^{-13} & 1.0 \times 10^{-13} & 5.3 \times 10^{-9} \\
\operatorname{Br}(\tau \rightarrow \mu \mu \mu): & 1.1 \times 10^{-13} & 2.1 \times 10^{-12} & 7.3 \times 10^{-13} & 1.7 \times 10^{-10} \\
\operatorname{Br}(\tau \rightarrow \text { eеe }): & 7.5 \times 10^{-15} & 3.2 \times 10^{-13} & 7.8 \times 10^{-15} & 3.9 \times 10^{-10}
\end{array}
$$

Again we have averaged over random lepton Yukawa couplings in the four different scenarios. In the result (5.36) we have only included the contribution of the $\mathrm{Z}$ boson exchange. The amplitude contributed by its first KK state is already suppressed by a factor of about twenty. We have included the first KK level of lepton states which is important in scenario (A) where the left-handed lepton positions are degenerate. The branching ratios depend on the KK scale as $1 / M_{K K}^{4}$. The experimental bound $\operatorname{Br}(\mu \rightarrow$ eee $)<1.0 \times 10^{-12}[29]$ is satisfied for leptons localized towards the Planckbrane and the rates can come close to the experimental sensitivity. It would be very interesting if a slow muon facility could test this process at the precision of $10^{-16}$ [30]. The scenario (B'), where the leptons are moved towards the TeV-brane, leads to strong flavor violation. The rate could only be brought down to the experimental bound if the KK scale is increased by an order of magnitude to about $100 \mathrm{TeV}$. The expected rates for tau decays are in all cases well below the latest Belle results $\operatorname{Br}(\tau \rightarrow \mu \mu \mu)<3.8 \times 10^{-7}$ and $\operatorname{Br}(\tau \rightarrow e e e)<2.7 \times 10^{-7}[31]$.

Severe experimental bounds have been put on $\mu e$-conversion in muonic atoms. The best exclusion limit $\operatorname{Br}(\mu N \rightarrow e N) \equiv \Gamma(\mu N \rightarrow e N) / \Gamma\left(\mu N \rightarrow \nu_{\mu} N^{\prime}\right)<6.1 \times$ $10^{-13}$ comes from the Sindrum-II collaboration [32]. We expect a branching ratio of

(A)

$$
\operatorname{Br}(\mu N \rightarrow e N): 5.0 \times 10^{-16}
$$

$5.2 \times 10^{-15}$
$\left(\mathrm{B}^{\prime}\right)$

$$
1.0 \times 10^{-15} 5.8 \times 10^{-11} \text {. }
$$

While the scenario (B') is again excluded for a $\mathrm{KK}$ scale of $10 \mathrm{TeV}$, leptons close towards the Planck-brane easily avoid the experimental limit. The forthcoming MECO experiment (E940 at BNL) plans to increase the sensitivity to about $5 \times 10^{-17}$ [33]. It would completely cover the predicted interaction rates, even for leptons close to the Planck-brane. 
At the one-loop level radiative decays that violate lepton flavor are induced at a rate

(A)

$$
\begin{array}{lll}
\operatorname{Br}(\mu \rightarrow e \gamma): & 2.1 \times 10^{-16} & 1.9 \times 10^{-15} \\
\operatorname{Br}(\tau \rightarrow \mu \gamma): & 6.7 \times 10^{-16} & 9.5 \times 10^{-15} \\
\operatorname{Br}(\tau \rightarrow e \gamma): & 2.8 \times 10^{-17} & 1.3 \times 10^{-15}
\end{array}
$$

(B)

$4.1 \times 10^{-16} 2.1 \times 10^{-11}$

$4.2 \times 10^{-15}$

$6.6 \times 10^{-13}$

$3.3 \times 10^{-17}$

Except for the scenario (B') the expected branching ratios are far below the experimental bounds $\operatorname{Br}(\mu \rightarrow e \gamma)<1.2 \times 10^{-11}, \operatorname{Br}(\tau \rightarrow \mu \gamma)<1.1 \times 10^{-6}$ and $\operatorname{Br}(\tau \rightarrow e \gamma)<2.7 \times 10^{-6}$. The MEG collaboration at PSI plans to increase the sensitivity with respect to $\mu \rightarrow e \gamma$ to about $10^{-13}-10^{-14}$ within the next years [34]. This will not be sufficient to test the model for leptons localized towards the Planck-brane. Note that our model leads to predictions quite different from supersymmetric models, where radiative decays occur at much larger rates than the decays of eqs. (5.36) and (5.37) [35]. In the warped SM radiative decays occur only at the loop level, while the latter are tree-level processes. In the scenario of Dirac neutrino masses radiative decays are also mediated by the KK states of the sterile neutrinos. If the SM neutrinos are confined to the TeV-brane, a too large branching ratio for $\mu \rightarrow e \gamma$ pushes the $\mathrm{KK}$ scale up to $25 \mathrm{TeV}$ and thus imposes a stringent constraint on the model [15]. However, the rate for $\mu \rightarrow e \gamma$ is sensitive to the mixing between light and heavy neutrino states. With bulk neutrinos the mixing with heavy states is considerably reduced. In ref. [10] a branching ratio $\operatorname{Br}(\mu \rightarrow e \gamma) \approx 10^{-15}$ was found for leptons localized towards the Planck-brane $(c>1 / 2)$. While this value is still well below the experimental sensitivity, it is larger than the contribution from gauge boson exchange (5.38). Other lepton flavor violating processes, such as muonium-antimuonium oscillations [36], do not lead to new constraints.

\subsection{Meson mass splittings and CP violation}

The flavor violating gauge couplings (5.31) also contribute to the mass splittings in neutral pseudo-scalar meson systems. The mass splitting between the flavor eigenstates of a meson, $P^{0}$ and $\bar{P}^{0}$, is given by

$$
\Delta m_{P}=\frac{\operatorname{Re}\left\langle P^{0}\left|-\mathcal{L}_{\mathrm{FCNC}}\right| \bar{P}^{0}\right\rangle}{m_{K}}
$$

where $\mathcal{L}_{\mathrm{FCNC}}$ contains the flavor violating couplings $(5.31)$. Phases in the flavor violation couplings are constrained by the indirect $\mathrm{CP}$-violation in the kaon system

$$
\epsilon_{K}=\frac{\operatorname{Im}\left\langle P^{0}\left|-\mathcal{L}_{\mathrm{FCNC}}\right| \bar{P}^{0}\right\rangle}{2 \sqrt{2} m_{K} \Delta m_{H}} .
$$

These expressions can be evaluated using the vacuum insertion approximation (see for instance [37]). The largest contributions to (5.39) and (5.40) come from the 
exchange of KK gluons [38]. Excited gluons have flavor non-universal couplings to fermions like the KK states of the weak gauge bosons and induce flavor violating couplings analogous to those of eq. (5.31). The gluon zero modes couple universally and therefore do not mediate flavor changing interactions.

For the quark locations of eq. (3.24) we obtain the following contributions of first level of $\mathrm{KK}$ gluons and the $\mathrm{Z}$ boson zero mode

$$
\begin{array}{rccc} 
& \text { gluon } & \text { Z } & \text { exp. } \\
\Delta m_{K}: & 1.5 \times 10^{-14} & 1.2 \times 10^{-17} & 3.5 \times 10^{-12} \\
\Delta m_{B}: & 5.1 \times 10^{-11} & 3.0 \times 10^{-14} & 3.2 \times 10^{-10} \\
\Delta m_{D}: & 3.8 \times 10^{-13} & 5.2 \times 10^{-15} & 4.6 \times 10^{-11} \\
\epsilon_{K}: & 1.1 \times 10^{-3} & 1.1 \times 10^{-6} & 2.3 \times 10^{-3} .
\end{array}
$$

The meson mass splittings are given in units of $\mathrm{MeV}$. In eq. (5.41) we give results averaged over random sets of Yukawa couplings $2 / 3<\left|l_{i j}\right|<4 / 3$. As in the case of flat extra dimensions [38] the dominating contributions come from the exchange of $\mathrm{KK}$ gluons. KK states of the $\mathrm{Z}$ boson give only an about ten percent correction to the zero mode exchange. In the computation of the fermion mass eigenstates we have included the first level of KK states. Compared to using only fermion zero modes the gluon contribution to $\epsilon_{K}$ is enhanced by a factor of two. The meson mass splittings are less sensitive to whether or not KK fermions are included. The neglect of higher KK levels amounts to an uncertainty of order unity in our results.

The contributions to meson mass splittings we find are much smaller than the experimental values [24] listed in the last column of eq. (5.41). The KK gluon contribution to $\epsilon_{K}$ comes rather close to the observed value. About one third of the random sets of Yukawa couplings we tested gave $\epsilon_{K}>2.3 \times 10^{-3}$, which is still acceptable. In ref. [17] KK contributions to $b \rightarrow s \gamma$ were considered. For the quark locations of eq. (3.24) a bound on the $\mathrm{KK}$ scale of about $5 \mathrm{TeV}$ was found. From the the process $K^{+} \rightarrow \pi^{+} \nu \bar{\nu}$ the limit on the Z coupling $\left|\mathcal{X}_{L, 1,2}^{d(0)}\right|<5.1 \times 10^{-6}$ was obtained in ref. [18]. We find a considerably lower value of $\left\langle\left|\mathcal{X}_{L, 1,2}^{d(0)}\right|\right\rangle=2.2 \times 10^{-7}$. Our approach to fermion masses and mixings is therefore nicely compatible with experimental constraints on flavor violation.

We stress that our results are very different from models where the fermions mass pattern is explained by flavor-dependent fermion locations in one or more universal flat extra dimensions. There flavor violation, in particular kaon mixing, leads to very restrictive bounds on the $\mathrm{KK}$ scale, $M_{K K} \gtrsim 10^{3} \mathrm{TeV}$ [38], disfavoring these models as a solution to the the gauge hierarchy problem. Recently it was shown that models constructed from intersecting D-branes suffer from a similar problem [39]. The crucial difference lies in the wave functions of the KK gauge bosons. With a warped extra dimension the gauge boson wave functions are almost constant away from the TeV-brane. Therefore the gauge couplings of KK gauge bosons are nearly universal for fermions localized somewhat towards the Planck-brane $(c>1 / 2)$ as 
we show in fig. 3. Flavor violation is thus automatically suppressed for the light fermion species which are expected to reside closely towards the Planck-brane to explain their small masses.

\section{Non-renormalizable operators and rare processes}

In models where the weak scale is identified with the fundamental scale of gravity, the low cut-off scale dramatically amplifies the impact of non-renormalizable operators in weak scale interactions. As a consequence, large rates for rare processes, such as flavor violation and proton decay, are a challenge for model building. With bulk fermions localized towards the Planck-brane the corresponding suppression scales can be significantly enhanced without relying on ad-hoc symmetries [5, 6]. However, there are limits because the SM fermions need to have sufficient overlap with the Higgs field at the TeV-brane to acquire their observed masses, as discussed in section 3. We consider the following generic four-fermion operators which are relevant for flavor violation as well as for proton decay

$$
\int d^{4} x \int d y \sqrt{-g} \frac{1}{M_{5}^{3}} \bar{\Psi}_{i} \Psi_{j} \bar{\Psi}_{k} \Psi_{l} \equiv \int d^{4} x \frac{1}{Q^{2}} \bar{\Psi}_{i}^{(0)} \Psi_{j}^{(0)} \bar{\Psi}_{k}^{(0)} \Psi_{l}^{(0)}
$$

Integrating over the extra dimension, the effective 4D suppression scales $Q$ associated with these operators depend on where the relevant fermion states are localized in the extra dimension.

Let us focus on some examples. The lepton flavor violating decay $\mu \rightarrow$ eee is induced by the operator $\mu$ eee at a rate $\Gamma \sim m_{\mu}^{5} / Q^{4}$. The experimental constraint on the corresponding branching ratio translates into $Q>5 \times 10^{5} \mathrm{GeV}$. For the lepton locations considered in the previous section we obtain the suppression scales

$$
\begin{array}{ccccc}
(\mathrm{A}) & \left(\mathrm{A}^{\prime}\right) & (\mathrm{B}) & \left(\mathrm{B}^{\prime}\right) \\
Q(\text { нeеe })[\mathrm{GeV}]: & 4.8 \times 10^{6} & 1.1 \times 10^{7} & 7.9 \times 10^{7} & 2.4 \times 10^{5},
\end{array}
$$

where we have taken all the leptons to be left-handed. Except for the case (B') where the leptons are localized towards the TeV-brane, the operator $\mu e e e$ is safely suppressed. The same holds for similar lepton flavor violating operators such as $\tau \mu \mu \mu$ and $\tau e e e$. The operator $\mu e q_{1} q_{1}$ contributing to muon electron conversion is constrained by $Q>1 \times 10^{5} \mathrm{GeV}$ while we are finding

$$
\begin{array}{ccccc}
(\mathrm{A}) & \left(\mathrm{A}^{\prime}\right) & (\mathrm{B}) & \left(\mathrm{B}^{\prime}\right) \\
Q\left(\mu e q_{1} q_{1}\right)[\mathrm{GeV}]: & 4.4 \times 10^{7} & 3.5 \times 10^{7} & 1.1 \times 10^{8} & 6.6 \times 10^{6} .
\end{array}
$$

These suppressions scales even exceed $Q>1 \times 10^{6} \mathrm{GeV}$, a bound which could be set by the upcoming MECO experiment. Thus non-renormalizable operators are not expected to induce lepton flavor violation at an observable rate, unless the leptons would be localized closely towards the TeV-brane. 
Constraints on the $K-\bar{K}$ mass splitting require the dimension-six operator $(d s)^{2}$ to be suppressed by $Q>5 \times 10^{6} \mathrm{GeV}$. If it contributes to $\mathrm{CP}$ violation, an even stronger suppression of $Q>5 \times 10^{7} \mathrm{GeV}$ is required. Using the quark locations of eq. (3.24), we obtain $Q>7.2 \times 10^{7} \mathrm{GeV}$ for left-handed and $Q>1.2 \times 10^{8} \mathrm{GeV}$ for right-handed states. For the operator $(d b)^{2}$ we find $Q>3.3 \times 10^{6} \mathrm{GeV}$, which is above the corresponding experimental bound of $2 \times 10^{6} \mathrm{GeV}$. Other flavor violating operators like $(\mathrm{cu})^{2}$ are also within their experimental bounds. The quark locations of eq. (3.24) which we obtained from the fermion mass pattern automatically lead to the required suppression of flavor violating non-renormalizable operators. This conforms the conclusions reached in refs. $[5,6]$.

Dimension-six operators contributing to proton decay are highly constrained by experimental searches. For instance the operator $q_{1} q_{1} q_{2} l_{3}$ has to be suppressed by $Q>10^{15} \mathrm{GeV}$ [40]. Taking again the quark locations of eq. (3.24) and the lepton locations of eq. (3.26) we obtain $Q>5.6 \times 10^{7} \mathrm{GeV}$. So some small coupling of order $10^{-14}$ should multiply the non-renormalizable operator to be consistent with experimental limits on proton decay. This might point to an additional symmetry, such as baryon or lepton number. A tiny coupling might also originate from nonperturbative effects of gravity, especially if there is an extra dimension somewhat larger than $M_{\mathrm{PL}}$ [41]. If the proton is stabilized by imposing lepton number (or lepton parity in the case of Majorana neutrino masses), baryon number violating processes like neutron-antineutron oscillations could still occur [42]. With the quark locations of eq. (3.24) the rate is, however, far below the experimental bound.

In ref. [6] somewhat different quark locations where used in order to maximally suppress proton decay by non-renormalizable interactions. Similar conclusions were reached, even though the suppression scale $Q$ could be enhanced by an order of magnitude. Non-renormalizable interactions can be further suppressed if the fermions are shifted closer towards the Planck-brane by allowing for larger 5D Yukawa couplings. A smaller AdS curvature compared to the fundamental Planck mass has the same effect. However, using these means to stabilize the proton would necessarily introduce new hierarchies in the model parameters.

\section{Conclusions}

In this paper we have studied aspects of flavor physics in the warped SM. Gauge bosons and fermions are bulk fields, while the Higgs field is confined to the $\mathrm{TeV}$ brane. We have shown in detail how the fermion mass hierarchies and mixings can naturally be explained in a geometrical way, without relying on hierarchical Yukawa couplings. The observed fermion masses and mixings fix the relative positions of the fermion fields in the extra dimension. Large mixings are attributed to similar locations, and masses become small for fermions localized towards the Planck-brane. Small neutrino masses can arise from a coupling to right-handed neutrinos in the bulk or from dimension-five interactions. 
Mixings between different KK levels induces deviations from the SM. Electroweak fits require the KK scale to be at least $10 \mathrm{TeV}$. KK mixings in the quark and weak gauge boson sectors lead to a non-unitary CKM matrix. However, deviations from unitarity are safely within the experimental bounds.

Flavor violation by $(\mathrm{KK})$ gauge boson exchange is an immediate consequence of our approach to the fermion mass problem. After transformation to fermion mass eigenstates, non-universal gauge couplings generate flavor changing neutral currents. Fixing the fermion locations by the fermion masses and mixings, we can predict the rates of flavor violations processes. Since the light fermion flavors are localized towards the Planck-brane, where non-universality is small, flavor violation is within experimental bounds even for a $\mathrm{KK}$ scale of $10 \mathrm{TeV}$. Some processes, such as muon-electron conversion, are in the reach of next generation experiments and can provide valuable hints to the higher dimensional theory. This result is quite different from models with flat extra dimensions, where constraints on kaon mixing require the $\mathrm{KK}$ scale to be in the $10^{3} \mathrm{TeV}$ range. Flavor violation in radiative decays is suppressed which is an important distinction to supersymmetric models.

Bulk fermions also help to reduce the impact of non-renormalizable operators. We have demonstrated that their contribution to flavor violating processes is naturally below the experimental sensitivity, even for order unity couplings. Dimensionsix operators leading to proton decay, however, cannot be sufficiently suppressed. The required tiny couplings might point to some additional symmetry.

Thus, the warped SM allows to generate the fermion mass pattern from flavordependent locations without giving up the solution to the gauge hierarchy problem or inducing unacceptable rates for flavor violating processes.

\section{Acknowledgements}

The author thanks David E. Costa and Qaisar Shafi for valuable discussions.

\section{References}

[1] L. Randall and R. Sundrum, Phys. Rev. Lett. 83 (1999) 3370.

[2] M. Gogberashvili, hep-ph/9812296.

[3] S. Chang, J. Hisano, H. Nakano, N. Okada and Yamaguchi, Phys. Rev. D62 (2000) 084025 [hep-ph/9912498].

[4] S.J. Huber and Q. Shafi, Phys. Rev. D63 (2001) 045010 [hep-ph/0005286].

[5] T. Gherghetta and A. Pomarol, Nucl. Phys. B586 (2000) 141 [hep-ph/0003129].

[6] S.J. Huber and Q. Shafi, Phys. Lett. B498 (2001) 256 [hep-ph/0010196]. 
[7] N. Arkani-Hamed and M. Schmaltz, Phys. Rev. D61 (2000) 033005 [hepph/9903417]; G.R. Dvali and M.A. Shifman, Phys. Lett. B475 (2000) 295 [hep$\mathrm{ph} / 0001072]$.

[8] S.J. Huber and Q. Shafi Phys. Lett. B544 (2002) 295 [hep-ph/0205327].

[9] Y. Grossman and M. Neubert, Phys. Lett. B474 (2000) 361 [hep-ph/9912408].

[10] S.J. Huber and Q. Shafi, Phys. Lett. B512 (2001) 365 [hep-ph/0104293].

[11] S.J. Huber, C.-A. Lee and Q. Shafi, Phys. Lett. B531 (2002) 112 [hep$\mathrm{ph} / 0111465]$.

[12] J.L. Hewett, F.J. Petriello and T.G. Rizzo, JHEP 0209 (2002) 030 [hep$\mathrm{ph} / 0203091]$.

[13] H. Davoudiasl, J.L. Hewett and T.G. Rizzo, hep-ph/0212279; M. Carena, E. Ponton, T.M.P. Tait and C.E.M. Wagner, hep-ph/0212307.

[14] C. Csaki, J. Erlich and J. Terning Phys. Rev. D66 (2002) 064021 [hep$\mathrm{ph} / 0203034$.

[15] R. Kitano, Phys. Lett. B481 (2000) 39 [hep-ph/0002279].

[16] F. del Aguila and J. Santiago, Phys. Lett. B493 (2000) 175 [hep-ph/0008143].

[17] C.S. Kim, J.D. Kim and J. Song, Phys. Rev. D67 (2003) 015001 [hep$\mathrm{ph} / 0204002]$.

[18] G. Burdman, Phys. Rev. D66 (2002) 076003 [hep-ph/0205329].

[19] S.J. Huber, to appear in the Proceedings of SUSY 2002, hep-ph/0211056.

[20] H. Davoudiasl, J.L. Hewett and T.G. Rizzo, Phys. Lett. B473 (2000) 43; A. Pomarol, Phys. Lett. B486 (2000) 153.

[21] H.B. Nielsen and Y. Takanishi, Phys. Lett. B543 (2002) 249 [hep-ph/0205180]; C.D. Froggatt, H.B. Nielsen and Y. Takanishi, Nucl. Phys. B631 (2002) 285 [hep-ph/0201152].

[22] H. Fusaoka and Y. Koide, Phys. Rev. D57 (1998) 3986-4001 [hep-ph/9712201].

[23] H. Fritzsch and Z. Xing, Prog. Part. Nucl. Phys. 45 (2000) 1 [hep-ph/9912358].

[24] K. Hagiwara et al. [Particle Data Group], Phys. Rev. D66 (2002) 010001.

[25] C. Jarlskog, Phys. Rev. Lett. 55 (1985) 1039.

[26] P.Q. Hung and M. Seco, hep-ph/0111013. 
[27] M. Beneke et al., hep-ph/0003033.

[28] P. Langacker and M. Plümacher, Phys. Rev. D62 (2000) 013006 [hep$\mathrm{ph} / 0001204]$.

[29] U. Bellgardt et al. [SINDRUM Collaboration] Nucl. Phys. B299 (1988) 1.

[30] J. Aysto et al., hep-ph/0109217.

[31] Y. Yusa et al., [BELLE Collaboration], hep-ex/0211017.

[32] P. Wintz, in Proceedings of the first International Symposium on Lepton and Baryon Number Violation, p. 534 (1998).

[33] M. Bachmann er al., [MECO Collaboration], Research Proposal E940 for an experiment at BNL (1997).

[34] L.M. Barkov, et al., [MEGA Collaboration], Research Proposal for an experiment at PSI (1999).

[35] J. Hisano and D. Nomura, Phys. Rev. D59 (1999) 116005 [hep-ph/9810479]; J.A. Casas and A. Ibarra, Nucl. Phys. B618 (2001) 171-204 [hep-ph/0103065].

[36] L. Willmann et al., Phys. Rev. Lett. 82 (1999) 49 [hep-ex/9807011].

[37] F. Gabbiani, E. Gabrielli, A. Masiero and L. Silvestrini Nucl. Phys. B477 (1996) 321 [hep-ph/9604387]; T. Moroi, JHEP 0003 (2000) 019 [hep$\mathrm{ph} / 0002208]$.

[38] A. Delgado, A. Pomarol and M. Quiros JHEP 0001 (2000) 030 [hep$\mathrm{ph} / 9911252]$.

[39] S. Abel, M. Masip and J. Santiago, hep-ph/0303087.

[40] K. Benakli and S. Davidson, Phys. Rev. D60 (1999) 025004 [hep-ph/9810280].

[41] A.B. Kobakhidze, Phys. Lett. B514 (2001) 131-138 [hep-ph/0102323]; R. Kallosh, A.D. Linde, D.A. Linde and L. Susskind, Phys. Rev. D52 (1995) 912.

[42] C.E. Carlson and C.D. Carone, Phys. Lett. B512 (2001) 121 [hep-ph/0103180]. 